This document is the accepted manuscript version of the following article:

Lees, J. M., Toumpanaki, E., Barbezat, M., \& Terrasi, G. P. (2017). Mechanical and durability screening test methods for cylindrical CFRP prestressing tendons. Journal of Composites for Construction, 21(2), 04016080 (13 pp.).

https://doi .org/10.1061/(ASCE)CC.1943-5614.0000727

\title{
Mechanical and durability screening test methods for cylindrical CFRP prestressing tendons
}

Janet M. Lees ${ }^{1}$, Eleni Toumpanaki ${ }^{2}$, Michel Barbezat ${ }^{3}$ and Giovanni P. Terrasi ${ }^{4}$

${ }^{1}$ Reader in Civil Engineering, University of Cambridge, Department of Engineering, Trumpington Street, Cambridge, CB2 1PZ, UK

${ }^{2}$ Foster and Partners, 22 Hester Rd, London, SW11 4AN, UK: Former PhD student, University of Cambridge, Department of Engineering, Trumpington Street, Cambridge, CB2 1PZ, UK

${ }^{3}$ Group Leader Composites, Mechanical Systems Engineering, Empa, The Swiss Federal Laboratories for Materials Science and Technology, Überlandstrasse 129, 8600 Dübendorf, Switzerland

${ }^{4}$ Head, Department of Mechanical Systems Engineering, Empa, The Swiss Federal Laboratories for Materials Science and Technology, Überlandstrasse 129, 8600 Dübendorf, Switzerland

\begin{abstract}
Concrete prestressed with carbon fibre reinforced polymer (CFRP) tendons beneficially utilises the strain capacity and durability characteristics of the CFRP material. However, changes to CFRP tendon material formulations or production processes present a challenge when building up a body of knowledge to inform the long-term behaviour of a prestressed concrete system. Initial qualification tests can help identify the potential for FRP tendons to meet long-term mechanical and durability requirements. Protocols that can be undertaken on commercially produced cylindrical FRP pultrusions are particularly advantageous since the constituent materials and manufacturing processes are representative of the actual product. Selected mechanical/durability characterisation approaches, and their suitability for
\end{abstract}


adaptation for wet specimens with cylindrical geometries, were critically assessed. A series of qualification tests were then conducted on CFRP tendons with three different resin systems. Tendon samples were exposed to wet environments to evaluate the longer-term solution uptake and associated mechanical durability implications. While characterisation measures such as the glass transition temperature, optical imaging and moisture uptake provided comparative results, the correlation with mechanical properties obtained from uniaxial tension, double notch shear and torsion tests was unclear. Using a sub-set of the mechanical test protocols, a retrospective analysis of CFRP tendons extracted from a prestressed concrete lighting pole under sustained load for 16.5 years was also reported. Subject Headings: Composite materials, Test procedures, Durability, Stress

\section{Introduction}

Civil infrastructure often requires design lives of 50 or more years. For prestressed concrete applications, high strength, high stiffness and durable reinforcing materials are necessary. Advanced composite carbon fibre reinforced polymer (CFRP) tendons offer a number of advantages over more traditional steel prestressing tendons. One of the strengths, and also the weaknesses, of CFRPs is that there are a multitude of variables that can be modified in order to achieve particular material properties. The use of different fibres, matrices, processing methods and parameters, surface finishes or coatings will all lead to different product outcomes. Fibre, resin and composite product systems are generally proprietary and end users often have little control over the implementation of changes in the material formulations even though these changes may impact the intended performance of the supplied materials. Yet, it is perhaps almost inevitable that products will change with time as a result of drivers such as material developments, economics and sustainability. But if the 
constituent materials or products evolve it is then unclear if earlier research work remains valid. This presents an obstacle for companies looking to use innovative CFRP systems.

These issues are particularly important in prestressed concrete applications where the CFRP tendons are highly stressed, potentially exposed to demanding environments and expected to maintain their structural integrity over a lifetime that extends to decades. The aim of the current work was to evaluate a series of relatively short-term tests as a means of quality control and/or for the initial long-term durability assessment of new candidate systems for CFRP pre-tensioned concrete. Mechanical performance metrics that are relevant to force transfer and the long-term durability in wet environments are of particular interest.

Cylindrical pultruded CFRP tendons with three different thermoset resin formulations are assessed. Initial characterisation tests are carried out on unexposed tendons to identify the glass transition temperatures and the presence of any internal voids. Uni-axial tendon tensile strengths are measured using either cast anchorage or clamped wedge anchorages. Exposed specimens are used to measure the moisture uptake in the tendons and double notch shear (DNS) and torsion tests are undertaken to identify potential changes in the matrix dominated mechanical behaviour of tendons due to exposure to wet environments. Tendons extracted from a prestressed concrete pole that had been highly stressed for 16.5 years (Terrasi et al. 2014) provide the context for a discussion of the connection between mechanical/durability tests and the observed in-service behaviour.

\section{CFRP Pultruded Tendons}

In CFRP prestressed concrete applications materials with a high strength and stiffness are required which implies a high fibre volume fraction (typically $>0.5$ ). Hence cylindrical 
pultrusions are preferred for use as prestressed tendons due to the unidirectionality of the fibres in the manufacturing process. Specifications for composites in civil engineering structural systems have been emerging e.g. Bank et al (2003). However, existing FRP standards tend to have a bias towards laminate structures based on the extensive research undertaken in naval and aerospace engineering. Failure modes in laminate structures can differ considerably from pultruded CFRP systems due to the weaker epoxy layer preferentially developed between laminas.

Test samples representative of unidirectional pultrusions are very difficult to create in laboratory conditions without a pultrusion production line. Neither vacuum bagging and/or pressure or vacuum injection moulding will replicate the high temperature/pressure and specific matrix flow conditions prevailing in a pultrusion die. Hence layered laminate test specimens designed to mimic pultrusions with similar fibre/resin constituents cannot reproduce the pultrusion curing cycle. Furthermore, common additives used in pultrusion such as internal release agents and calcium carbonate (1-2 $\mu \mathrm{m}$ in diameter) can not necessarily be incorporated in laboratory production processes as they can lead to incompatibilities within the matrix system and induce phase separation and precipitation (Empa (2009)). So while it is possible to produce a laminate sample with the same fibre and resin systems as a FRP tendon, the properties of the test specimen will differ from the pultruded product since the surface finishing, curing cycle, lamination etc. will not be the same. This is a significant limitation since the importance of the manufacturing process on the mechanical properties and durability of FRP rods has been highlighted by several authors (Benmokrane and Mohamed (2013); Toumpanaki et al. (2013)). The manufacturing process affects the degree of crosslinking in the epoxy e.g. due to curing time and temperature, and the presence and percentage of voids e.g. due to die length and heat power, pull out speed. 
In the current work, the mechanical/durability assessment focuses on approaches that can be adapted/undertaken on commercially pultruded cylindrical rods. This represents the best way for the results to be representative of the actual materials used in practice. A comprehensive picture is garnered by combining insights gained from material characterisation observations, mechanical testing and long-term durability studies where matrix dominated shear effects are explored in the context of solution exposure.

\section{CFRP Tendons for Prestressed Concrete Applications}

Prestressed tendon or cable systems are likely to consist of a series of small diameter cables e.g. between 4 and $6 \mathrm{~mm}$. Smaller tendon diameters are desirable to create slender elements, reduce anchorage lengths and mitigate prestress transfer splitting and transverse thermal expansion effects. As a result, the tendon diameters can fall outside the remit of existing standards that specifically relate to pultruded rods e.g. ASTM D4476-09 (2009). In addition, the initial tendon prestress is often $50-60 \%$ of the ultimate tendon failure load and so throughout the lifetime of a structure the tendon will sustain a force in excess of $50 \%$ of its breaking stress. Therefore creep and relaxation are also important design considerations.

A specific requirement for prestressed concrete with bonded tendons is the need to transfer force through interface shear both in the fabrication and in-service stages. In pretensioned concrete structures, the tendons need to be anchored temporarily during concrete casting and initial curing. There must also be sufficient bond between the tendon and concrete in the concrete anchorage zones so that when the stress is transferred, the transfer length is not excessive, this being an area of the structural element in which the shear and bending load carrying capacity is limited by the development of the precompression (Bruggeling (2001), 
Terrasi et al (2012)). When placed in-service and subjected to applied loads, the tendon force to an extent depends on whether the concrete cracks. If concrete cracking occurs, there will be a local concentration of tendon stress at the crack locations and this increase will be transferred to the concrete through bond. Excessive bond will lead to a lack of deformability (Lees and Burgoyne (1999)) but sufficient bond is required to ensure small cracks and force transfer between the FRP and concrete. To improve this transfer, the tendons may have an additional surface sand coating to improve bond (Terrasi (2013)).

The in-service exposure conditions of a prestressed concrete structure can be harsh. In outdoor applications, even in European climates the surface temperature of the concrete cover of a CFRP tendon prestressed concrete pole exposed to sun has been measured to be as high as $60^{\circ} \mathrm{C}$ (Terrasi et al (2011b)). The internal relative humidity in high performance concrete can reach $87 \%$ (Terrasi et al (2012)). Prestressed concrete structures are typically designed to be fully prestressed in service but water can move through the concrete cover from the exposure face. If the concrete cracks under particular service or extreme load combinations the tendons will be further exposed to wet conditions. Furthermore, salt water exposure is common in marine environments and highway structures subjected to deicing salts. These conditions need to be reflected in initial screening tests and one priority is how to combine relatively short-term exposure regimes with mechanical and physical testing that will then be indicative of the long-term in-service performance. In terms of the long-term durability, carbon fibres have generally been found to have very good durability properties (see Mantell (1968)). However, the epoxy matrix will absorb moisture with time which may influence matrix-dominated properties such as the bond and tendon shear stiffness and strength (Toumpanaki et al. 2015). Exposure to moisture is likely to have a greater impact on mechanical properties that are dependent on the matrix properties. 


\section{Test protocols and application to cylindrical pultrusions}

In the following, a general discussion of the material characterisation of CFRP cylindrical pultrusions namely $T_{g}$, optical microscopy, moisture uptake observations; uni-axial tension testing and short beam shear tests is presented. Modifications required for cylinders, and the potential, or lack thereof, for the methods to capture changes due to exposure to moisture is highlighted. Further test protocols suitable for adaptation for wet specimens with cylindrical geometries that have not been commonly used in the literature, nor combined with moisture, namely double notch shear and torsion tests, are then critically assessed.

\section{Optical microscopy}

Optical microscopy can be used as a simple and direct quality control procedure for pultruded CFRP rods to visually assess the fibre distribution and the presence of any voids. Voids have been observed in commercially available FRP rods (Davalos et al. (2008)) suggesting a need for better quality controls. An increase in the moisture uptake in CFRP rods with voids has been reported (Toumpanaki et al. (2015)) and voids can aggravate the expected strength reduction of composites under hygrothermal conditions (Zhang et al. (2010), Costa et al. (2004)). An increase in the void content results in a decrease mainly in the matrix dominated mechanical properties, e.g. ILSS (Bowles and Frimpong (1992)), and there is also a dependency on the void geometry and shape.

\section{Glass transition temperature}

The glass transition temperature can potentially give an indication of the extent of curing and the performance in service. An epoxy with a high glass transition temperature, $T_{g}$, is advantageous to mitigate any reduction in properties when exposed to higher temperatures. 
But such a material may be more brittle and difficult to anchor (Terrasi et al (2011a)). A related consideration is the desirability of a high impact toughness to mitigate the possibility of accidental tendon damage during handling operations in the prestressing plant. A standard method to achieve this is by toughening the epoxy resin through the addition of microscopic rubber particles. But the presence of these particles has been found to lead to a reduction of the glass transition temperature (Giannakopoulos et al (2011)). A minimum $T_{g}$ for carbon FRP products with $V_{f}>0.5$ has been proposed to be $\geq 93.3^{\circ} \mathrm{C}$ (Bank et al (2003)). However, the value of $T_{g}$ depends significantly on the method of measurement and the $T_{g}$ definition used. The glass transition temperature is typically measured using either DMTA (Dynamic mechanical thermal analysis) or DSC (Differential scanning calorimetry) (Sims (2007)).

In exposed samples, the initial moisture content will vary and this will influence the measured $T_{g}$. Wright (1981) proposed a 'rough rule-of-thumb' suggesting a $1 \%$ increase in moisture content leads to a decrease in $T_{g}$ of around $20^{\circ} \mathrm{C}$. The effect of moisture on the $T_{g}$ value has been highlighted in standards (e.g. ASTM D7028, 2008). Wright (1981) also noted that for different resins the water absorption at saturation may differ by a factor of ten and, for a given resin, variations in the curing cycle can lead to differences of a factor of three.

\section{Moisture Uptake}

An indication of the potential for the matrix to absorb moisture over time is to submerse unstressed cylindrical tendon specimens in water and weigh them periodically. This does not directly replicate the combined in-service exposure and tendon stress conditions. However, it is difficult to obtain consistent and repetitive mass uptake readings of stressed and exposed CFRP tendons while under accelerated ageing conditions. 
Fickian approaches can be used to describe the moisture uptake in polymeric materials.

Diffusion models that reflect relaxation-dependency in epoxies have also been investigated (Glaskova et al, 2007). Fickian models have previously been applied to study the moisture uptake in composites (Bao and Yee (2002)). The presence of fibres affects the moisture transport and knock-down factors based on assumed packing arrays have been incorporated to model fibre influences e.g. (Keller and Sachs, 1963). As a relative measure, it is useful to compare the Fickian parameters for FRP tendons. A diffusion model based on Fickian behaviour is dependent on two inherent material variables; the mass at saturation $M_{\text {sat }}$ and the radial diffusion rate $D$ through a cylindrical rod. This can be described in Equation 1 as

$\frac{M_{t}}{M_{s a t}}=1-\sum_{n=1}^{\infty} \frac{4}{r_{o}^{2} a_{n}^{2}} \exp \left(-D a_{n}^{2} t\right)$

where $M_{t}$ is the mass uptake at time $t, r_{o}$ is the tendon radius and $a_{n}$ are the zero roots of the Bessel function. Assuming radial diffusion dominates, the Fickian diffusion rate, $D$, can be also calculated from the gradient of the experimental mass uptake $M_{t}$ versus $\sqrt{t}_{t}$ plot where:

$\frac{M_{t}}{M_{s a t}}=\frac{4}{\sqrt{\pi}}\left(\frac{D t}{r_{o}^{2}}\right)^{1 / 2}$

However, Crank (1975) noted that the simplified form of equation 2 for Fickian mass uptake behaviour is more valid when $M_{t} \leq 0.6 M_{\text {sat }}$ and is not as robust for cylinders as for the equivalent expression for plane sheets. Therefore, in the current work, an iterative process based on Equation 1 will be applied to find the diffusion coefficient by using the immersion time and the respective mass uptake reading $M_{t}$ as input variables.

\section{Uniaxial tension}

In terms of strength measures, the determination of the uniaxial tensile strength of a tendon is an important part of a qualification regime for use as FRP reinforcement and prestressing. The tensile strength of a tendon is typically a fibre-dominated property. Researchers have 
undertaken tensile tests on cylindrical putrusions after exposure to solutions to assess any degradation in the tensile strength (e.g. Uomoto, 2001). The difficulty with exposed tensile tests is that, unless the ends are protected, it is impossible to separate the anchorage and exposure effects since any changes in matrix properties will also affect the anchorage. Indeed, Bank et al (2003) noted that the testing of exposed tendons in tension may exhibit a matrix failure in the gripping region leading to erroneous lower tensile strength values (due to a reduction in the load transfer mechanism from matrix to fibres.). In certain cases, there may even be a beneficial influence of exposure possibly due to the deterioration of the matrix properties thereby reducing the propensity for shear lag (Scott \& Lees 2012). However, in absence of matrix effects, the degradation of the tensile strength in FRP rods due to solution uptake is not expected to be significant since the fibres dominate the failure mode. Studies have shown almost no decrease in tensile strength of exposed CFRP tendons in concrete pore solution (Micelli and Nanni (2004), Chen et al. (2007)).

\section{Shear properties}

The in-plane shear strength and interface shear properties tend to be either matrix-dominated, or fibre-matrix interphase dominated since fibre oxidation, sizing and alignment can have a considerable influence on mechanical properties e.g. ILSS. These properties are therefore susceptible to solution exposure. Transverse shear, double notch shear (DNS), and torsion testing approaches were investigated to determine their potential to ascertain the influence of solution exposure on the shear-related properties of cylindrical pultruded specimens.

\section{Transverse shear}

Transverse shear tests have been carried out on exposed GFRP (e.g. Gentry (2011)) or CFRP tendons (e.g. Scott and Lees (2012)). Unstressed cylindrical CFRP specimens (Scott and 
Lees (2012)) were loaded transversely with flat plates after exposure to $60{ }^{\circ} \mathrm{C}$ water, salt water or concrete pore solutions for 540 days. The peak failure loads of the specimens in water were similar to the unexposed control specimens. However, an average of a $4 \%$ or $7 \%$ reduction in failure load was noted in specimens exposed to salt solution or concrete pore solution respectively. Differences in stiffness were also observed. Scott and Lees (2012) also undertook short beam tests with rounded loading plates to negate the local stress concentrations induced by the sharp edges of the flat plates. For specimens loaded with rounded plates, a decrease in the delamination stresses in the range of 25-27\% was observed after exposure to water or salt water immersion conditions. Greater reductions were noted in the concrete pore solution tests. Bank et al (2003) propose short beam testing after 28, 56, 112 and 224 days of conditioning to assess the interlaminar (ILSS) shear of an FRP tendon. In an elastic beam in bending, the maximum shear stress is at the neutral axis. However, in a circular tendon, unless fully saturated, the moisture profile will potentially vary along the neutral axis of the beam.

\section{Double Notch Shear}

A transverse shear test and a double notch shear (DNS) test measure somewhat different phenomena and each has its strengths and weaknesses. The DNS test is a representation of the interlaminar shear strength and can be adapted to test circular profiles. However, the test has been found to be very sensitive to machine and notch geometry (Shokrieh and Lessard (1998)) and high local stress concentrations are generated at the notches (Pettersson and Neumeister (2006)). Recent studies have also shown that DNS performed in tension or in compression can lead to different results (Empa unpublished results) and this is assumed to be due to the different stress concentrations around the notch when loaded in tension or in compression. Since a central plane is being tested, this test will be most meaningful for 
exposed specimens when the specimens are fairly well saturated, as in the case of ILSS.

Otherwise, the moisture profile may be non-uniform along the shear plane of interest.

\section{Torsion testing}

Circular CFRP tendons are well-suited to torsion testing. In exposed CFRP tendons the outer tendon surface saturates most quickly and this is the surface that sustains the highest stress in a torsion test. So the impact of any deterioration in the matrix stiffness should be apparent even after relatively short-term conditioning. Reductions in the shear modulus of $17 \%$ and $29 \%$ were recorded for two different cylindrical CFRP tendons systems after exposure in water for 141 days at $23^{\circ} \mathrm{C}$ and 71 days at $60^{\circ} \mathrm{C}$ (Toumpanaki et al., 2015). A predictive model where the shear modulus degradation was related to the concentration profile through the tendon captured the experimental trends (Toumpanaki et al., 2015).

\section{Experimental Materials and Methods}

The methods described in the previous section were benchmarked by comparing the performance of $5.4 \mathrm{~mm}$ diameter tendons with three different matrix systems, EPR 4434/943, EPR 4434/IPD and EPR4434/Nanopox/IPD. Further details of the material properties are shown in Table 1. The pultrusion cure cycle for all the tendons was 4 minutes at $180^{\circ} \mathrm{C}$ followed by a proprietary postcure regime. As produced, the tendons had an outer sand coating layer to enhance the bond between the tendon and concrete (Terrasi (2013)). For consistency, in some tests it was necessary to remove this sand coating layer. In the following, unless stated explicitly that the tendons were previously dried or immersed, the specimens for mechanical tests were conditioned for at least $72 \mathrm{~h}$ under normal climatic conditions $\left(23^{\circ} \mathrm{C} / 50 \%\right.$ relative humidity) prior to testing under the same conditions. 


\section{Optical Microscopy}

To detect whether voids were present and to study the fibre distribution, $5 \mathrm{~mm}$ long samples of the CFRP tendons were cut and prepared for optical microscopy pictures. The samples were cast into a resin matrix (Acri-kleer cold mounting) and polished with P400-P1200 abrasive silicon paper. The samples were studied using a Leica DMLM Optical Microscope (Leica Microsystems, Germany) under either a bright or dark field.

\section{Glass transition temperature $T_{g}$}

The glass transition temperature $T_{g}$ was measured using DMTA (Dynamic mechanical thermal analysis) and DSC (Differential scanning calorimetry). For the DMTA tests (Eplexor 500 from Gabo Qualimeter, Germany) under three point bending mode, the sand coating layer was smoothly scratched away from the original rods previously stored in lab conditions. A minimum of two samples were tested. The tests were performed on samples with a total length of $80 \mathrm{~mm}$, span of $60 \mathrm{~mm}$ and nominal diameter of $5.4 \mathrm{~mm}$ at a $10 \mathrm{~Hz}$ frequency and heating rate of $2^{\circ} \mathrm{C} / \mathrm{min}$ from $20^{\circ} \mathrm{C}$ to $210^{\circ} \mathrm{C}$. A dynamic amplitude of 0.015 $\%$ strain was superposed on a static load of $0.125 \%$ flexural strain. The DMTA $T_{g}$ values were calculated using either the onset of $\mathrm{E}^{\prime}$ (storage modulus) or the maximum of the damping factor $(\tan \delta)$ definition. The DSC experiments (DSC 7 from Perkin Elmer) were conducted at $20^{\circ} \mathrm{C} / \mathrm{min}$ from $50^{\circ} \mathrm{C}$ to $220^{\circ} \mathrm{C}$ on $50-70 \mathrm{mg}$ samples (without the sand coating layer) The half-Cp values were used to define $T_{g}$.

\section{Moisture uptake}

Unstressed specimens of the CFRP tendons studied were fully exposed in water . The outer sand coating layer was manually removed with a blade to reduce the variability in the mass uptake readings due to the detachment of the sand particles. However, the removal raises a 
question as to whether the outer surface has been damaged. On balance it was deemed preferable to remove the coating to obtain consistent results. The tendons were initially dried in an oven at $60^{\circ} \mathrm{C}$ but the drying times differed. The $4434 / 943$ specimens were dried for 2 years and a mass loss of $0.58 \%$ was noted. For the IPD samples, the drying times were either six months (with a recorded mass loss of $0.38 \%$ ), or ten months (with a mass loss of $0.40 \%$ ) for specimens subsequently exposed at either $23^{\circ} \mathrm{C}$ or $60^{\circ} \mathrm{C}$ respectively. The dessicated CFRP tendons were then immersed in water at $23^{\circ} \mathrm{C}$ and $60^{\circ} \mathrm{C}$ (to simulate accelerated aging conditions). Mass uptake readings were regularly recorded with an Oertling R20 (Oertling, U.K.) analog balance machine with a 0.0001 -g resolution to provide a basis for inferring the diffusion properties of the materials.

\section{Uniaxial tension}

Even with bespoke anchorage systems, when testing CFRP tendons in tension there is a high likelihood of a premature failure in the end of the tendon anchorage due to transverse compression and shear stresses. Conical cast anchorages and short-term tendon clamping anchorage systems were investigated. For the cast anchors, the total length of each tensile specimen was $700 \mathrm{~mm}$, which led to a free length of $400 \mathrm{~mm}$ between the $140 \mathrm{~mm}$ long cylindrical anchorage sockets. The crucial issue of gripping the tendons was solved by epoxy bonding high strength steel sockets (grade ETG 100) to both tendons ends. The CFRP tendon specimens were centred and fixed with a silicon drop at the load entrance of the steel anchorages, after having sprayed a separating PTFE layer on the anchorages' inner surface. A low viscosity lamination epoxy resin (Araldite LY 5052 (Huntsman, 2004)) was then cast in the conical interior of the cylindrical steel sockets (Terrasi, 1998). The steel anchorages were laterally fixed to steel adaptors, which were then clamped in the wedge adaptors of a 
universal servo-hydraulic Instron testing machine as shown in Fig. 1a. The test speed was 2 $\mathrm{mm} / \mathrm{min}$ and the strain was measured with a clamped mechanical extensometer.

Clamped wedge anchorage systems are reusable and quick to install. This is particularly advantageous for pre-tensioned concrete where it is only necessary to impart tension into the tendon until the concrete gains sufficient strength for the release of prestress. The system is described in detail in Terrasi et al. (2011a) and consisted of a conical steel anchorage with three wedges made of a thermoplastic polymer with short glass fibre reinforcement (see Fig. 1b). In the wedge anchorage tensile tests the total tendon length was $450 \mathrm{~mm}$ and the free length was $330 \mathrm{~mm}$. The rate of loading was $4 \mathrm{~mm} / \mathrm{min}$ and the strain measurement was derived from a clamped mechanical extensometer.

\section{Double Notch Shear (DNS)}

Baseline shear strengths were obtained from the DNS tension tests on specimens that had previously been stored in normal climate conditions $\left(23^{\circ} \mathrm{C} / 50 \%\right.$ rel. humidity). The DNS tests were undertaken in an analogous method to that of the DIN 65148 (1986) standard. The specimens had a total length of $200 \mathrm{~mm}$ with a shear length of $12.5 \mathrm{~mm}$ between 2 cuts machined with a rectangular shaped diamond coated circular saw. The central test area is supported/guided with a low friction anti-buckling device visible in Fig. 2. The tests were conducted at $0.5 \mathrm{~mm} / \mathrm{min}$ in a universal testing machine. These were not pre-dried so there will have been a level of moisture in the samples. The DNS results were compared with tests where the specimens were notched and then immersed in a salt water solution for seven months at $60^{\circ} \mathrm{C}$ prior to testing.

Torsion testing (matrix stiffness) 
The principles of the ASTM E143-02(2008) and conditioning were combined to develop a screening test for the matrix stiffness. Torsion tests (see Fig. 3) were carried out on samples that had been immersed in water at $23^{\circ} \mathrm{C}$ or $60^{\circ} \mathrm{C}$. In these tests, a length of tendon was gripped at one end. A lever arm was then clamped to the tendon a set distance away from the grip and weights were applied through the lever arm. Inclinometers were used to measure the relative rotation at each load increment, hence the torsional stiffness response could be measured. The stiffness was measured as an average from three load unload cycles for either one (EPR 4434/943 and EPR 4434/Nanopox/IPD), or two (EPR 4434/IPD) tendon samples. Control samples were tested under dry conditions. Exposed samples were tested at a theoretical Fickian mass uptake $M_{t} \approx 0.54 \%$ at either $23^{\circ} \mathrm{C}$ or $60^{\circ} \mathrm{C}$ and by assuming $M_{t}=M_{\text {sat }}$ after 1240 days at $23^{\circ} \mathrm{C}$.

\section{Results and Discussion}

\section{Optical Microscopy}

In Fig. 4, optical microscopy pictures from a EPR 4434/943 rod sample are shown relative to a $1000 \mu \mathrm{m}$ (Fig. 4a), $200 \mu \mathrm{m}$ (Fig. 4b) and $50 \mu \mathrm{m}$ (Fig. 4c \& d) scale. There appears to be a solid matrix distribution with no voids. The only abnormalities are small areas of different 'glassy' morphology that are more pronounced in Fig. 4d, where a dark field is applied for comparison. Fig. 5 shows a cross-section of an EPR 4434/IPD CFRP rod. A localised void of roughly $300 \mu \mathrm{m}$ is observed near the surface of the sample. Fig. 5c at a scale of $200 \mu \mathrm{m}$ reveals a non-uniform fibre distribution with a denser distribution in the brighter areas. A non-uniform fibre distribution was also observed in a EPR4434/Nanopox/IPD rod (Fig. 6).

Glass transition temperature $T_{g}$ 
The results are reported in Table 2 . The DMTA E' onset $T_{g}$ values were fairly similar for all three products (between $121-125^{\circ} \mathrm{C}$ ) but when a $\tan \delta_{\max }$ definition was used the $T_{g}$ of the EPR $4434 / 943$ specimens was $10^{\circ} \mathrm{C}$ lower than the EPR 4434/IPD samples. The measured $T_{g}$ values using DSC ranged from 120 to $135^{\circ} \mathrm{C}$ and the EPR 4434/IPD results exhibited the greatest variability. The different hardeners and the presence of the filler appeared to have a small influence on the glass transition temperature but there were no clear trends.

Differences in the humidity of the samples may be contributing to variations in the results.

\section{Moisture uptake}

The mass uptake with time is shown in Fig. 7 . At $23^{\circ} \mathrm{C}$, the measured uptakes in the EPR 4434/943 and EPR 4434/IPD specimens were fairly similar but, at a given time, the uptake in the EPR 4434/Nanopox/IPD tendon was consistently lower. After nearly four years, the moisture uptake continues to increase but there appears to be a reduction in the slope which would be indicative of pending saturation. At $60^{\circ} \mathrm{C}$, all three tendon materials have fairly similar initial mass uptakes in the early stages (up to around 100 days). The EPR 4434/943 samples were exposed for a longer period than the other materials and exhibit a mass loss after 1-1.5 years of exposure at $60^{\circ} \mathrm{C}$ suggesting that the higher temperature and the longer exposure time promote matrix degradation. The EPR 4434/IPD specimens exhibited greater standard deviation in the mass uptake reading that could be attributed to the presence of the localised void as observed in the optical microscopy pictures. The geometrical characteristics of this void might vary along the CFRP tendon coil.

As discussed, an iterative process was implemented by using the immersion time and the respective mass uptake reading $M_{t}$ as input variables to then find the best-fit diffusion coefficient from Equation 1. Since the mass at saturation level was not yet forthcoming, 
purely for indicative purposes, $M_{\text {sat }}$ was assumed as the last mass uptake value after 1138 days ( 23.1 years) of immersion in water at $23{ }^{\circ} \mathrm{C}$. This then leads to calculated diffusion rates for the 4434/943, 4434/IPD and 4434/Nanopox/IPD tendons of $2.42 \times 10^{-10} \mathrm{~cm}^{2} / \mathrm{sec}, 3.02 \times 10^{-}$ ${ }^{10} \mathrm{~cm}^{2} / \mathrm{sec}$ and $2.34 \times 10^{-10} \mathrm{~cm}^{2} / \mathrm{sec}$ respectively. The predicted profiles using these diffusion rates for a number of different time intervals (28, 56 and 127 days) have been plotted in Fig. 8. Even after 127 days, there is significant non-uniformity in the predicted moisture profile. This has implications in terms of the time-frame for characterisation tests that would reflect any changes in the properties due to exposure.

\section{Tension testing}

Uni-axial tension tests were carried out using resin cast socket anchorages. The cast sockets visible in Fig. 1a can be either screwed into the machine or clamped. Failures occurred either in the tendon free length or inside the socket, possibly initiated at the end of the cone. The average tendon strengths and standard deviations obtained from testing 5-8 samples using the cast anchorage system are presented in Table 3. These were compared with the theoretical values of the tensile strengths of the tendons based on the number of CF-rovings (using a volume fraction of $67 \%$ for EPR 4434/943 and 68\% for the IPD systems), the fibre strength and an estimate of an average pultrusion efficiency of $90 \%$ based on the experience of the pultruder. The theoretical strength of the rods should be around $2900 \mathrm{MPa}$. However, the measured strengths using the resin cast anchors were found to be $22 \%$ to $31 \%$ lower than the theoretical tensile stress values. The highest average measured strength was $2262 \mathrm{MPa}$ for the EPR4434/Nanopox/IPD tendons with cast anchors but these tendons also exhibited the greatest standard deviation. 
Tensile tests with clamped anchorages were also undertaken (Fig. 1b) and the results included in Table 3. The axial strength results using clamped anchorages are consistently lower than the cast anchor results and ranged between 1468 and $1695 \mathrm{MPa}$. The reduction is postulated to be due to local compressive and shear stress concentrations in the anchor (Terrasi et al (2011a)). Although the cast anchorage strength of the Nanopox material had been the highest, the clamped anchorage strength was similar to the 4434/IPD. This suggests that the stress states in the clamped and cast anchors may differ. To characterise the sensitivity of the tendon to clamping forces and inform the design of more efficient clamping geometries, transverse compression tests between two flat plates without sand or two curved plates with sand were undertaken (Terrasi et al (2011a)). In these transverse compression tests (see Fig. 9), the samples failed due to combined shear and transverse tension. The measured transverse compressive strength for the flat and curved plates (Table 4) showed that the EPR 4434/Nanopox/IPD samples had the highest transverse compression strength. The EPR 4434/IPD flat plate results without sand had the highest standard deviation. The connection between the transverse compression results and anchorage strengths is inconclusive.

\section{Double notch shear tests}

The DNS results are shown in Table 5 where the average measured value from 5 samples is presented. The associated mass uptakes $\left(7\right.$ months at $60^{\circ} \mathrm{C}$ in salt water) at the time of testing are also presented. When lab-conditioned, the filled EPR4434/Nanopox/IPD matrix had an improved performance when compared with the unfilled resins. However, the EPR 4434/943 and EPR 4434/IPD specimens were 15-19\% weaker after immersion whereas losses of 28\% were noted in the EPR4434/Nanopox/IPD tendons. Hence the post-immersion DNS results of the three materials were similar. The measured strengths did not seem to correlate with the mass uptake since the Nanopox/IPD system had the lowest salt water uptake but greatest 
reduction in DNS. Any solution induced mechanisms that impact the fiber / matrix interface and/or induce local plasticization of the matrix could dominate the DNS behaviour, especially as the rectangular notch promotes solution access to the cut regions sustaining stress concentrations and this would not necessarily be captured in the moisture uptake in the bulk CFRP tendon material. Two phase epoxy structures are also preferentially developed at the fiber-matrix interphase (Vanlandigham et al. 1999) and this can be more dominant in the Nanopox/IPD systems due to the agglomeration of the nanoparticles.

\section{Torsion tests}

The 'dry' torsion test results and the results after exposure to water have been summarised in Table 6. Exposed specimens were tested at specified mass uptakes of around $0.5 \%(0.51 \%$ or $0.54 \%$ ) to provide a common basis for comparison. The shear modulus values after 1240 days exposure are also presented. Even though the predicted moisture profiles (see Fig. 8c) are similar, the $60^{\circ} \mathrm{C} 4434 / \mathrm{IPD}$ samples showed a greater loss in stiffness than the equivalent $23^{\circ} \mathrm{C}$ specimens at $0.54 \%$ uptake suggesting temperature causes a marginally greater degradation. The $23^{\circ} \mathrm{C}$ stiffness reduction of the 4434/943 and IPD specimens after 1240 days was slightly greater than that of the samples tested after 141 or 127 days ( $-8 \%$ vs $-11 \%$ and $+1 \%$ vs $-4 \%$ ), whereas the EPR4434/Nanopox reductions were similar after 266 and 1240 days of exposure at $23^{\circ} \mathrm{C}$. The lower shear modulus of EPR 4434/IPD dry samples might be attributed to the void area. The voids in EPR 4434/IPD could also lead to a higher variability in the torsion test results and a higher degradation at $60^{\circ} \mathrm{C}$. Therefore, these factors may be contributing to the differences in the EPR $4434 / \mathrm{IPD} 23^{\circ} \mathrm{C}$ and $60^{\circ} \mathrm{C}$ stiffness results.

\section{Evaluation of test protocols and case study of long-term in-service performance}


The direct evaluation of cylindrical pultrusions overcomes unrepresentative limitations introduced when trying to manufacture analogous laminate samples by hand in the laboratory. This is a significant advantage. The current work demonstrated that it was possible to apply/adapt a range of test protocols to suit cylindrical pultrusions. However, when solution uptake is incorporated to investigate durability influences, the non-uniform nature of the concentration profile at a given time needs to be addressed in the context of the property being measured.

Optical imaging proved to be a powerful means to identify voids in a sample and provide insight into the fibre distribution. This is advantageous for quality control. However, unless there are visible signs of deterioration e.g. due to moisture exposure, this method will not reveal indications of detrimental effects on the mechanical properties. The glass transition temperature is a standard measure but dependent on the test set-up and definition used, and the initial moisture content in the tendons. Moisture uptake gives a relative baseline measure to compare different products but correlations with mechanical properties require further development. Furthermore, even when the exposure takes place under elevated temperatures, the timeframes to saturation can be lengthy thereby limiting the effectiveness for short-term qualification purposes. The uni-axial tensile strength is an important metric but tensile tests are fundamentally challenging due to anchorage issues. It is thus difficult to quantify the influence of moisture exposure on the tensile strength. DNS samples require careful machining and the fibres can influence the response of the failure plane. The DNS tests did give an indication of strength changes due to moisture exposure. However, unless saturated, the moisture profile through the cylindrical section is non-uniform so the moisture level across the failure plane can vary. Transverse shear tests including short-beam shear tests are widely used but mixed-modee stresses and fibre-stiffening effects are potential issues. There 
is also a non-uniformity of the moisture profile through the beam cross-section to be taken into account in a durability assessment. Torsion tests were readily suited to cylindrical specimens and were effective in assessing matrix stiffness changes due to exposure. Further work is required to determine the viability of torsion testing to measure the matrix strength.

An opportunity arose to evaluate selected methodologies in the context of a retrospective analysis of $3 \mathrm{~mm}$ diameter CFRP tendons from a prototype prestressed concrete lighting pole section that had been under sustained load for 16.5 years. Unstressed tendon material stored in the laboratory for a similar period was also available. When considering the matrix of possible tests, moisture uptake testing was discounted since the failed tendons had already been subjected to a typical external environment. But it is of note that most of the length of the tendon samples removed from the concrete were not in the vicinity of a crack and therefore not directly exposed at a crack face. Since the tendon diameter was only $3 \mathrm{~mm}$, transverse shear and DNS testing were difficult to undertake with confidence. The focus therefore was on uni-axial tension and torsion testing.

\section{CFRP prestressed high strength concrete pole}

The high strength pole specimen was manufactured by SACAC Ltd and had a wall thickness of 25-27 mm with eight CFRP tendons distributed radially around the central perimeter. The concrete was centrifugally-cast with a minimum cube strength of $115 \mathrm{MPa}$ after 28 days. The $3 \mathrm{~mm}$ diameter CFRP tendons were coated with aluminium oxide sand. The pole specimen was left outside under a sustained loading (Fig. 10 shows the associated loading arrangement and the failed pole) that induced a stress in the lowest tendon of around $2460 \mathrm{MPa}$ at the beginning of testing. Through stress redistribution in the pole this tendon stress increased to approximately $2940 \mathrm{MPa}$ in 15 years. After 16.5 years under a load equivalent to $72 \%$ of the 
short term bending failure load, the pole specimen failed. Full details of the test programme and results can be found elsewhere (Terrasi et al, 2014). Intact sections of the tendons were carefully removed from the broken concrete pole and used as test specimens.

\section{Uniaxial tensile testing}

Samples of the same tendon material that had been stored at SACAC Ltd for 18 years in an unstressed $2 \mathrm{~m}$ coil in uncontrolled laboratory conditions (approximately $15-25^{\circ} \mathrm{C}, 40-70 \%$ relative humidity) were tested as control specimens. Previous tensile test results from 1995 on equivalent $3 \mathrm{~mm}$ pultruded tendons from the same batch without sand coating (Terrasi 1998) were also used as comparators. The strain up to failure was monitored in the 1995 tests with an optical extensometer and the measured Young's modulus (secant modulus between 0.5 and $1 \%$ strain), failure stress and failure strain were reported as $180.7 \pm 2.7 \mathrm{GPa}, 3375 \pm$ $136 \mathrm{MPa}$ and $1.87 \pm 0.07 \%$ respectively.

The samples extracted from the failed pole and the material stored in the laboratory were tested in the same manner and with the same cast anchorage system as the original tests from 1995. However, for both sets of aged samples, the failure mode changed from the tendon failures in the free length observed in 1995 to failures in the socket at the end of the anchorage at average stresses of $2436 \pm 33 \mathrm{MPa}$ for the stored tendons and $2515 \pm 228 \mathrm{MPa}$ for the failed pole samples (see Fig. 11). Subsequent changes were made in the preparation of the anchorage to try to eliminate anchorage failures but these were without success. It is believed that either the aged samples have lost part of their transverse compressive and/or shear strength inducing premature anchorage failure or the potting resin system used for casting the anchorage has changed. The anchorage resin is nominally the identical 
formulation to that used in 1995 but changes in the products cannot be excluded. A stiffer cast anchorage could induce higher local stress concentrations and induce premature failure.

In the 2015 tests, a mechanical extensometer captured the strain up to about $70-80 \%$ of the expected ultimate load and was removed prior to the (premature) failures. The elastic secant moduli derived from strains between 0.5 and $1 \%$ led to a modulus of $179 \pm 0.5 \mathrm{GPa}$ for the specimens stored in the lab and $186 \pm 3 \mathrm{GPa}$ for the failed pole specimens. The average elastic modulus of the stored tendons was therefore similar to the 1995 values but, rather surprisingly, the elastic modulus of the tendons extracted from the failed pole was $4 \%$ and more than two standard deviations higher (see Fig. 11). A possible explanation would be some minor alignment effects of the carbon fibres due to matrix creep under prestress and sustained loading of 16.5 years. This effect would be consistent with the higher Young's modulus measured in the extracted specimens.

\section{Torsion testing}

Torsion testing was conducted on three tendon samples extracted from the failed pole and also three samples from the material stored in lab conditions. Adjustments to the torsion rig shown in Fig. 3 were required to accommodate the smaller diameter tendons. In particular, the diameter of the clamping ring attachment and the weight of the loading lever arm were reduced. The torsion results are presented in Table 7. The measured stiffness of the samples from the pole after long-term sustained loading was on average $4 \%$ higher than the lab samples. This is consistent with the trends observed in the elastic modulus results (see Fig. 11) where the pole samples were also stiffer with the caveat that the axial modulus is dominated by fibre effects. Using lab storage as a baseline reference it appears that exposure to the concrete alkaline environment was not detrimental. The lower shear modulus of the 
material from the coil in the lab could be connected to the storage conditions since the tendons were uncovered and next to a window. In particular UV light is known to deteriorate the epoxy matrix of CFRP and so the tendons in the pole might have been protected by the concrete cover in this respect. Also, some creep effects during storage as a coil may contribute to the weakening of the tendons when straightened during testing.

\section{Conclusions}

Various test methods were critically assessed for their suitability for use with representative cylindrical pultrusions and incorporation of solution exposure. Three CFRP tendons with different resin systems, EPR 4434/943, EPR 4434/IPD and EPR 4434/Nanopox/IPD, were compared across selected characterisation and mechanical qualification tests with and without solution exposure. Optical imaging indicated voids in the EPR 4434/IPD tendons and nonuniform zones of fibres in both the IPD and Nanopox/IPD tendons. The glass transition temperature results were found to be inconclusive and depended on the measurement method used and the definition of $T_{g}$. The EPR4434/Nanopox/IPD moisture uptake was lower than that of the EPR 4434/943 and EPR 4434/IPD tendons at $23^{\circ} \mathrm{C}$. After reaching a peak, the $4434 / 943$ material experienced a loss of mass at $60^{\circ} \mathrm{C}$ suggesting that degradation processes not observed at $23^{\circ} \mathrm{C}$ could occur after exposure to water at $60^{\circ} \mathrm{C}$. Prior to saturation, the concentration profile in a cylindrical specimen is non-uniform and, depending of the test method, this has implications in terms of the moisture conditions across a test plane. In general, the determination of the uni-axial tensile strength is hampered by difficulties associated with premature tendon failures in the anchorages. Reductions of between $17 \%$ and $28 \%$ in the tendon DNS strengths were observed due to exposure to salt water solution for 7 months at $60^{\circ} \mathrm{C}$. The tendon torsional stiffnesses generally decreased by $4 \%$ to $11 \%$ after moisture exposure. Considering the mechanical behaviour after moisture exposition and the 
corresponding test results, both local and/or bulk effects due to either plasticization of the matrix or damage of the fibre / matrix interface have to be taken into account in conjunction with moisture gradient effects discussed above.

While the various protocols allow for a comparison across different materials, the difficulty is ascertaining the connection with the long-term mechanical performance in-service. This challenge was highlighted in a retrospective analysis of tendons removed from a failed concrete pole that had been under sustained load for 16.5 years. Premature anchorage failures were observed in tensile tests. However, the tensile elastic modulus and torsional stiffness measurements suggested that the stiffnesses of the failed pole samples were marginally higher than equivalent samples stored in the laboratory.

\section{Acknowledgements}

The authors are very grateful to SACAC Ltd for their technical advice and financial assistance. The work was supported by Swiss CTI-Project 8465.1 EPRP-IW. Funding from the Onassis Foundation for one of the authors (ET) is also appreciated.

\section{References}

ASTM D4476 - 09 Standard Test Method for Flexural Properties of Fiber Reinforced Pultruded Plastic Rods, ASTM International.

ASTM D7028 - 07 Standard Test Method for Glass Transition Temperature (DMA Tg) of Polymer Matrix Composites by Dynamic Mechanical Analysis (DMA), ASTM International. ASTM E143 - 02(2008) Standard Test Method for Shear Modulus at Room Temperature, ASTM International. 
Bank, L.C., Gentry, T.R., Thompson, B. P. and Russell, J.S. (2003). A Model Specification for FRP Composites for Civil Engineering Structures, Construction and Building Materials 17(6-7), pp 405-437.

Bao, L.R. and Yee, A.F. (2002). Moisture diffusion and hygrothermal aging in bismaleimide matrix carbon fibre composites- part I: uni-weave composites. Composites Science and Technology, 62, pp. 2099-2110.

Benmokrane B. and Mohamed H.M. (2013). Durability Issues of FRP for Civil Infrastructure. In the $11^{\text {th }}$ International Symposium on Fiber Reinforced Polymers for Reinforced Concrete Structures (FRPRCS-11), Eds. J. Barros and J. Sena-Cruz, Universidade do Minho, Guimarães, Portugal,14 pp.

Bowles, K. J. and Frimpong, S. (1992). Void Effects on the Interlaminar Shear Strength of Unidirectional Graphite-Fiber-Reinforced Composites. Journal of Composite Materials 26(10), pp 1487-1509.

Bruggeling, A.S.G. (2001). Übertragen der Vorspannung mittels Verbund (Prestress Transfer by Bond). Beton und Stahlbetonbau (Concrete and Reinforced Concrete Construction), 96(3), pp 109-123.

Chen, Y., Davalos, J.F., Ray, I. and Kim, H-Y (2007). Accelerated Aging Tests for Evaluations of Durability Performance of FRP Reinforcing Bars for Concrete Structures. Composite Structures 78(1), pp 101-111.

Costa, M.L., Rezende, M.C. and Almeida, S.F.M. (2004). Strength of Hygrothermally Conditioned Polymer Composites with Voids. J. of Compos Mater, 39(21), 1943-1961. Crank (1975). The Mathematics of Diffusion, Clarendon Press, Oxford, UK. Davalos, J.F, Chen, Y. and Ray, I. (2008). Effect of FRP Bar Degradation on Interface Bond with High Strength Concrete. Cement and Concrete Composites, 30(8), pp 722-730. 
DIN 65148 (1986) standard: Prüfung von faserverstärkten Kunststoffen, Bestimmung der interlaminaren Scherfestigkeit im Zugversuch; Beuth Verlag GmbH, Berlin.

Empa (2009) CTI-Project 8465.1 EPRP-IW: New Ways of Increasing the Prestress of Filigree CFRP Prestressed High Performance Concrete Elements. (unpublished)

Gentry, T.R. (2011). Transverse Shear of GFRP rods: Tests Method Development and Potential for Durability Assessment. In Fourth International Conference on Durability and Sustainability of Fiber Reinforced Polymers (FRP) Composites for Construction and Rehabilitation, CDCC, 2011, Department of Civil Engineering Faculty of Engineering Université de Sherbrooke, Sherbrooke, Canada.

Giannakopoulos G., Masania, K., Taylor, A. C. (2011). Toughening of Epoxy Using CoreShell Particles, Journal of Material Science, 46, pp 327-338.

Glaskova, T.I., Guedes, R.M., Morais, J.J., and Aniskevich, A.N. (2007). A comparative analysis of moisture transport models as applied to an epoxy binder. Mechanics of Composite Materials, 43(4): p. 377-388.

Huntsman (2004), Advanced Materials data sheet: Co-curing epoxy system based on Araldite LY 5052 / Aradur 5052.

Keller, H.B. and D. Sachs (1964), Calculations of the Conductivity of a Medium Containing Cylindrical Inclusions. Journal of Applied Physics, 35(3): p. 537-538.

Lees, J.M. and Burgoyne C.J. (1999). Experimental Study of the Influence of Bond on the Flexural Behaviour of Concrete Beams Pre-tensioned with AFRPs, ACI Structural Journal, 96(3), May-June, pp 377-385.

Mantell, C.L. (1968). Carbon and Graphite Handbook, New York: John Wiley \& Sons. Micelli, F. and Nanni, A. (2004). Durability of FRP Rods for Concrete Structures, Construction and Building Materials, 18 (7) pp. 491-503. 
Pettersson, K.B. and Neumeister, J.M. (2006). A Tensile Setup for the IDNS Composite Shear Test, Compos Part A: Appl S, 37(2), pp 229-242.

Scott, P. And Lees, J.M. (2012). Effects of Solution Exposure on the Combined Axial-shear Behaviour of Unidirectional CFRP Rods, Compos Part A: Appl S, 43(9), pp 1599-1611.

Shokrieh, M.M. and Lessard, L.B (1998) An Assessment of the Double-Notch Shear Test for Interlaminar Shear Characterization of a Unidirectional Graphite/Epoxy under Static and Fatigue Loading, Applied Composite Materials 5: 289-304.

Sims, G. D. (2007), Review of Composites Standardisation Status and Activities, National Composites Group.

Terrasi, G.P. (1998), Mit Kohlenstoffasern vorgespannte Schleuderbetonrohre. Ph.D. Thesis, Eidgenössische Technische Hochschule (ETH), Zurich, Switzerland (In German) Terrasi, G.P. (2013), Prefabricated Thin-walled Structural Elements Made from High Performance Concrete Prestressed with CFRP Wires, Journal of Material Science Research 2(1), p. 1-14.

Terrasi, G.P., Affolter, C., and Barbezat, M. (2011a). Numerical Optimization of a Compact and Reusable Pre-tensioning Anchorage System for CFRP Tendons. J. Comp. for Constr., 15(2), Special Issue in Honor of Professor Urs Meier, pp 126-135.

Terrasi , G.P. Meier, U and Affolter, C (2014). Long-Term Bending Creep Behavior of ThinWalled CFRP Tendon Pretensioned Spun Concrete Poles. Polymers, 6, pp 2065-2081. Terrasi, G.P, Broenimann, R. Baettig, G. (2011b). CFRP Tendon Prestress Monitoring by Resistance Measurement in a High Performance Concrete Powerline Pylon. In Proceedings of SMAR 2011, the 1st Middle East Conference on Smart Monitoring, Assessment and Rehabilitation of Civil Structures : 8 - 10 February 2011, Dubai, UAE, Eds M. Motavalli, B. Havranek and E. Saqan, Empa, CH-8600 Dübendorf, Swizterland, 8 pp. 
Terrasi, G.P., Bisby, L., Barbezat, M., Affolter, C., and Hugi, E. (2012). Fire Behavior of Thin CFRP Pretensioned High-Strength Concrete Slabs. J Comp for Constr, 16(4), pp 381394.

Toumpanaki, E., Lees, J. M. and Terrasi, G.P. (2013). Effect of Moisture Uptake on the Shear Stiffness of CFRP Tendons and the Role of the Manufacturing Route, FRPRCS11, 11th International Symposium on Fiber Reinforced Polymer for Reinforced Concrete Structures. Eds. J. Barros and J. Sena-Cruz. Universidade do Minho, Guimarães, Portugal. Toumpanaki, E., Lees, J. M. and Terrasi, G.P. (2015). Shear Modulus of Cylindrical CFRP Tendons Exposed to Moisture. J Comp for Constr, 19(3) 04014059.

Uomoto, T. (2001). Durability Considerations for FRP Reinforcements. FRPRCS-5: Fibrereinforced Plastics for Reinforced Concrete Structures, Ed. C.J. Burgoyne, Volume 1, pp 1732, Thomas Telford, London, UK.

Vanlandingham, M.R., Eduljee, R.F., and Gillespie, J.W. (1999). Relationships between stoichiometry, microstructure, and properties for amine-cured epoxies. Journal of Applied Polymer Science, 71(5), 699-712.

Wright, W.W. (1981). The effect of diffusion of water into epoxy resins and their carbon-fibre reinforced composites. Composites, 12(3): p. 201-205.

Zhang, A.Y., Li, D.H., Zhang, D.X., Lu, H.B., Xiao, H.Y. and Jia, J. (2011). Qualitative Separation of the Effect of Voids on the Static Mechanical Properties of Hygrothermally Conditioned Carbon/Epoxy Composites. Express Polymer Letters, 5(8), pp 708-716. 
Table 1. Material properties

\begin{tabular}{lccc}
\hline Property & EPR4434/943 & EPR4434/IPD & $\begin{array}{c}\text { EPR4434/ } \\
\text { Nanopox/IPD }\end{array}$ \\
\hline Epoxy & Bisphenol A & Bisphenol A & Bisphenol A \\
Hardener & EPH 943 & IPD & IPD \\
Nanopox $\left(\mathrm{SiO}_{2}\right)$ & - & - & $5 \%$ \\
Mix ratio & $100 / 33$ & $100 / 33$ & $100 / 36.7 / 18.5$ \\
Fibre type & Tenax UTS50 & Tenax UTS50 & Tenax UTS50 \\
& F13-12K- & F24-24K- & F24-24K- \\
Fibre tensile strength & $800 t e x$ & 1600 tex & $1600 t e x$ \\
Fibre Young's Modulus & $5100 \mathrm{MPa}$ & $5000 \mathrm{MPa}$ & $5000 \mathrm{MPa}$ \\
Fibre tensile ultimate & $245 \mathrm{GPa}$ & $250 \mathrm{GPa}$ & $250 \mathrm{GPa}$ \\
strain & $2.1 \%$ & $2.0 \%$ & $2.0 \%$ \\
Volume fraction & & & \\
\hline
\end{tabular}

Table 2. Glass transition temperature $T_{g}$ from pultruded rod samples (single values with a typical reproducibility of $\pm 1-2^{\circ} \mathrm{C}$ )

\begin{tabular}{lccc}
\hline Tendon material & \multicolumn{3}{c}{$T_{g}{ }^{\circ} \mathrm{C}$} \\
\cline { 2 - 4 } & $\mathrm{DSC}$ & $\mathrm{DMTA}$ & DMTA \\
& $1 / 2 \mathrm{Cp}$ & $\mathrm{E}^{\prime}$ onset & $\tan \delta_{\max }$ \\
\hline EPR 4434/943 & $125-130$ & 121 & 150 \\
EPR 4434/IPD & $120-135$ & 125 & 160 \\
EPR4434/Nanopox/IPD & 130 & 125 & 155 \\
\hline
\end{tabular}

Table 3. Tensioning assessment (average and standard deviation of 5-8 samples)

\begin{tabular}{lccc}
\hline Tendon material & \multicolumn{3}{c}{ Strength (MPa) } \\
\cline { 2 - 4 } & Theoretical & $\begin{array}{c}\text { Cast } \\
\text { anchorage }\end{array}$ & $\begin{array}{c}\text { Clamped } \\
\text { anchorage }\end{array}$ \\
\hline EPR 4434/943 & 2851 & $1959^{* *}$ & $1468 \pm 76$ \\
EPR 4434/IPD & 2894 & $2066 \pm 64$ & $1695 \pm 70$ \\
EPR4434/Nanopox/IPD & 2894 & $2262 \pm 122$ & $1690 \pm 54$ \\
\hline
\end{tabular}

** Standard deviation not available 
Table 4. Transverse compression testing (average and standard deviation of 5-7 samples)

\begin{tabular}{lcc}
\hline Tendon material & \multicolumn{2}{c}{ Strength $(\mathrm{kN})$} \\
\cline { 2 - 3 } & $\begin{array}{c}\text { Flat plates } \\
\text { no sand }\end{array}$ & $\begin{array}{c}\text { Curved saddles } \\
\text { with sand }\end{array}$ \\
\hline EPR 4434/943 & $12.6 \pm 0.6$ & $20.1 \pm 1.3$ \\
EPR 4434/IPD & $11.4 \pm 3.6$ & $20.0 \pm 0.9$ \\
EPR4434/Nanopox/IPD & $14.7 \pm 0.4$ & $25.3 \pm 2.0$ \\
\hline
\end{tabular}

Table 5. Shear properties before / after salt water (SW) uptake 7 months @ 60

\begin{tabular}{lccc}
\hline Tendon material & Water uptake & \multicolumn{2}{c}{ DNS (MPa) } \\
\cline { 3 - 4 } & & original & $\begin{array}{c}\text { after SW } \\
\text { exposure }\end{array}$ \\
\hline EPR 4434/943 & 0.97 & $36 \pm 2$ & $30 \pm 2$ \\
EPR 4434/IPD & 1.13 & $37 \pm 3$ & $30 \pm 5$ \\
EPR4434/Nanopox/IPD & 0.92 & $43 \pm 2$ & $31 \pm 4$ \\
\hline
\end{tabular}

Table 6. Torsion results

\begin{tabular}{|c|c|c|c|c|c|c|}
\hline \multirow{3}{*}{ Tendon material } & \multicolumn{6}{|c|}{ Stiffness (GPa) } \\
\hline & \multirow{2}{*}{ 'Dry' } & \multicolumn{5}{|c|}{ After exposure } \\
\hline & & $\begin{array}{c}127 \text { days } \\
23^{\circ} \mathrm{C} \\
M_{t}=0.54 \%\end{array}$ & $\begin{array}{c}266 \text { days } \\
23^{\circ} \mathrm{C} \\
M_{t}=0.54 \%\end{array}$ & $\begin{array}{c}141 \text { days } \\
23^{\circ} \mathrm{C} \\
M_{t}=0.51 \%\end{array}$ & $\begin{array}{c}25 \text { days } \\
60^{\circ} \mathrm{C} \\
M_{t}=0.54 \%\end{array}$ & $\begin{array}{c}1240 \text { days } \\
23^{\circ} \mathrm{C} \\
M_{t} \approx M_{\infty}\end{array}$ \\
\hline EPR 4434/943 & $5.92 \pm 0.13$ & & & $\begin{array}{c}5.43 \pm 0.17 \\
(-8 \%)\end{array}$ & & $\begin{array}{c}5.26 \pm 0.02 \\
(-11 \%)\end{array}$ \\
\hline $\begin{array}{l}\text { EPR 4434/IPD } \\
\text { (series 1) }\end{array}$ & $5.52 \pm 0.05$ & $\begin{array}{c}5.56 \pm 0.06 \\
(+1 \%)\end{array}$ & & & $\begin{array}{c}5.29 \pm 0.07 \\
\quad(-4 \%)\end{array}$ & $\begin{array}{c}5.32 \pm 0.02 \\
(-4 \%)\end{array}$ \\
\hline $\begin{array}{l}\text { EPR 4434/IPD } \\
\text { (series 2) }\end{array}$ & $5.66 \pm 0.06$ & $\begin{array}{c}5.57 \pm 0.03 \\
(-2 \%)\end{array}$ & & & $\begin{array}{c}5.12 \pm 0.08 \\
(-10 \%)\end{array}$ & \\
\hline $\begin{array}{l}\text { EPR } 4434 / \text { Nano } \\
\text { pox/IPD }\end{array}$ & $5.81 \pm 0.04$ & & $\begin{array}{c}5.53 \pm 0.05 \\
(-5 \%)\end{array}$ & & $\begin{array}{c}5.41 \pm 0.04 \\
(-7 \%)\end{array}$ & $\begin{array}{c}5.44 \pm 0.01 \\
(-6 \%)\end{array}$ \\
\hline
\end{tabular}

Table 7. Torsional stiffness of $3 \mathrm{~mm}$ diameter tendons

\begin{tabular}{lccc}
\hline Sample & Specimen No & Stifness (GPa) & Average (GPa) \\
\hline Failed pole & 1 & $6.78 \pm 0.22$ & \\
& 2 & $7.42 \pm 0.03$ & $7.20 \pm 0.36$ \\
& 3 & $7.39 \pm 0.13$ & \\
\hline Coil stored in & 1 & $7.02 \pm 0.06$ & \\
lab & 2 & $7.14 \pm 0.06$ & $6.91 \pm 0.18$ \\
& 3 & $6.75 \pm 0.08$ & \\
& 4 & $6.72 \pm 0.02$ & \\
\hline
\end{tabular}




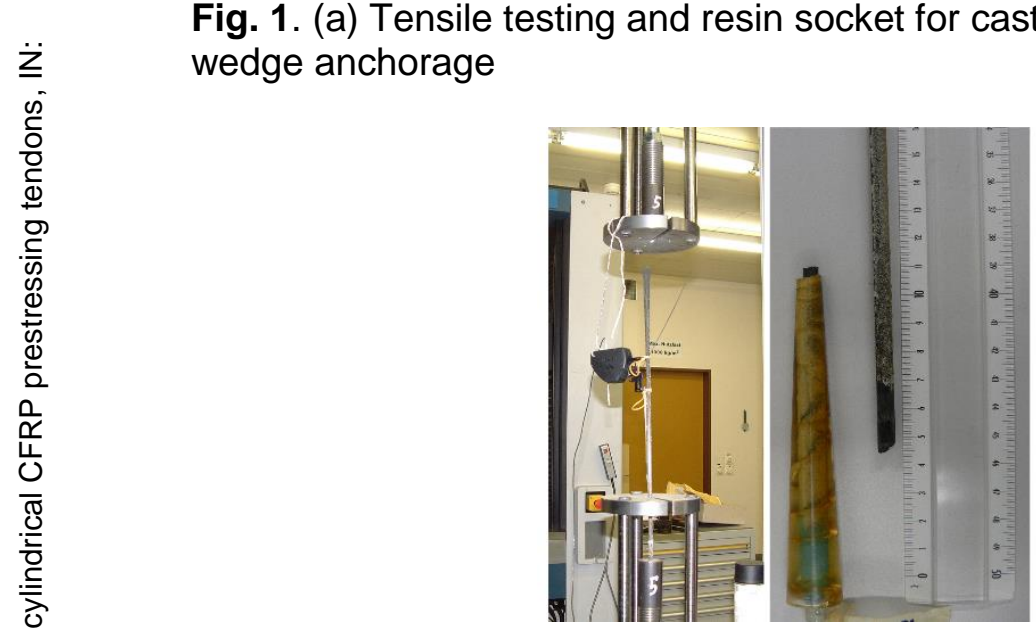

(a)

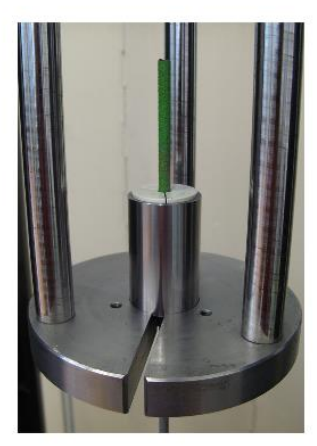

(b)

Fig. 2. Double notch shear testing of tendon samples adapted from DIN 65148 standard
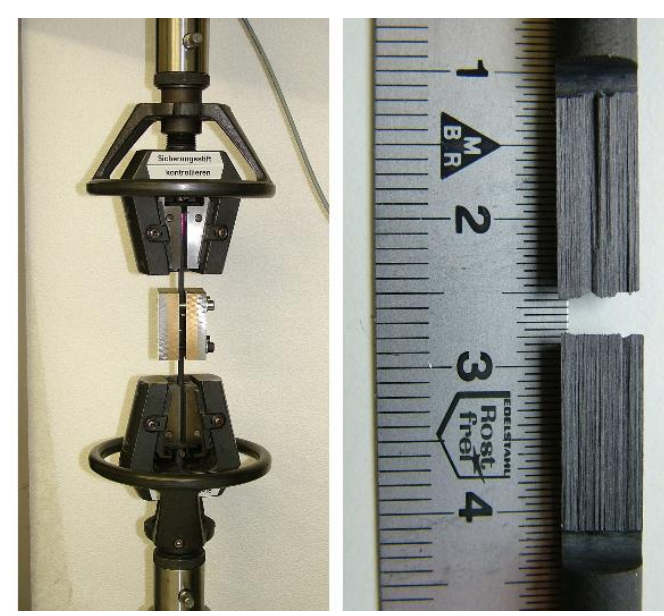

Fig. 3. Torsion testing of CFRP tendons

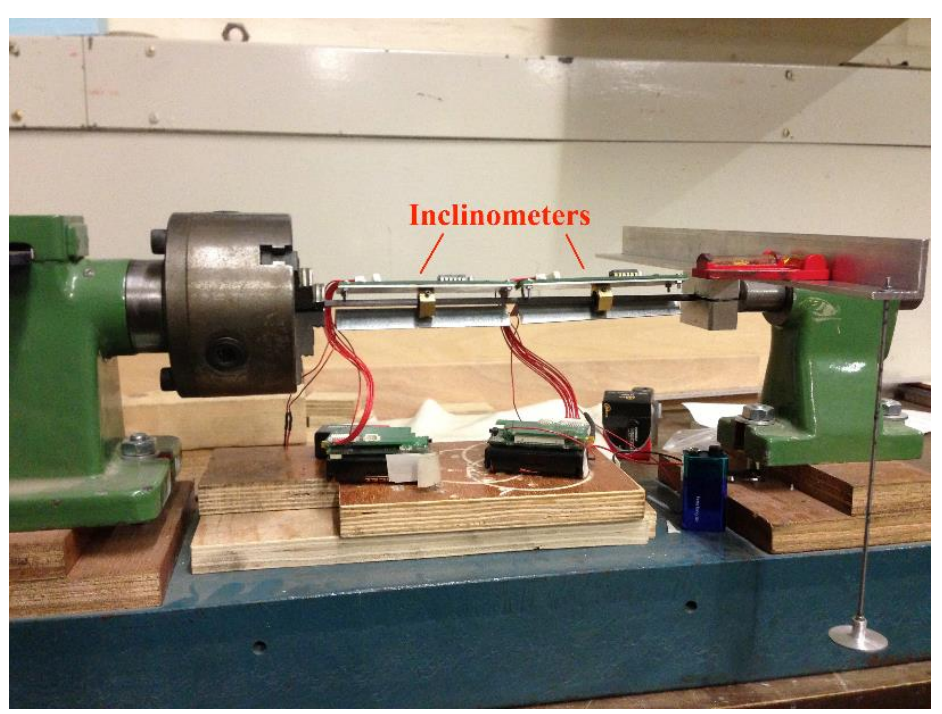


Fig. 4. EPR $4434 / 943$ (a) $1000 \mu \mathrm{m}$ (b) $200 \mu \mathrm{m}$ (c) $50 \mu \mathrm{m}$ and (d) $50 \mu \mathrm{m}$ (with a dark field)

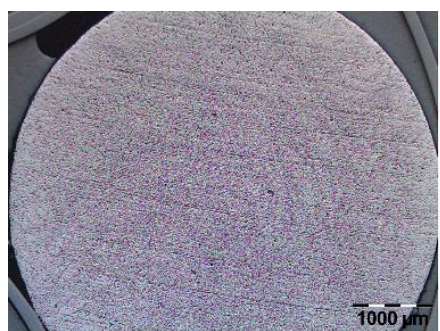

(a)

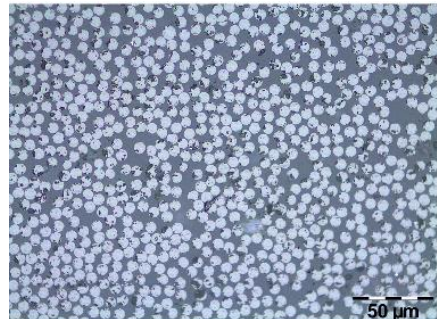

(c)

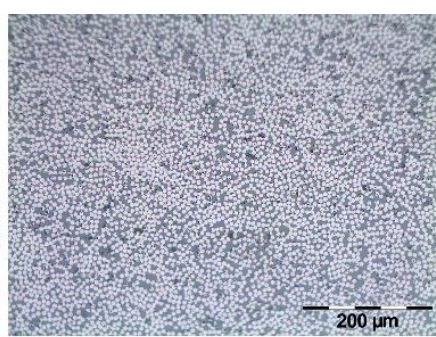

(b)

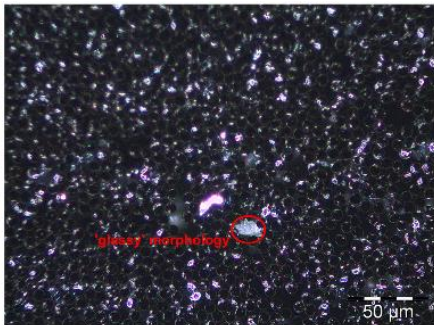

(d)

Fig. 5. EPR 4434/IPD (a) heterogeneity $1000 \mu \mathrm{m}$ (b) void $1000 \mu \mathrm{m}$ (c) heterogeneity $200 \mu \mathrm{m}$ (d) void $200 \mu \mathrm{m}$

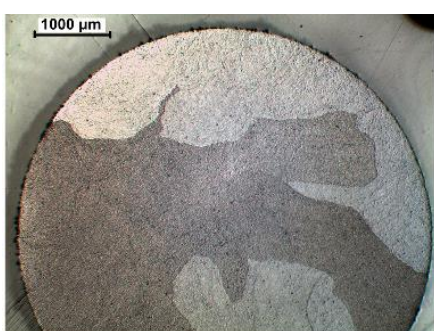

(a)

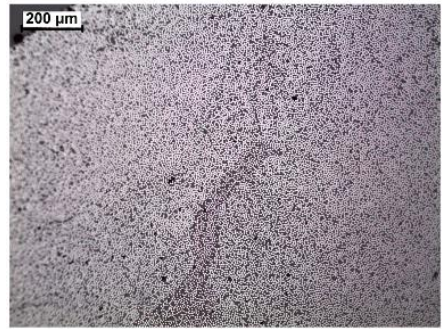

(c)

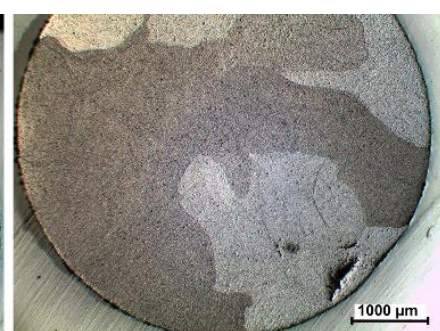

(b)

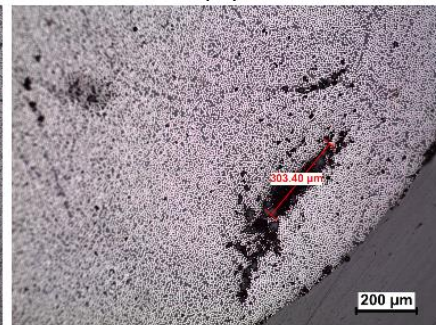

(d) 


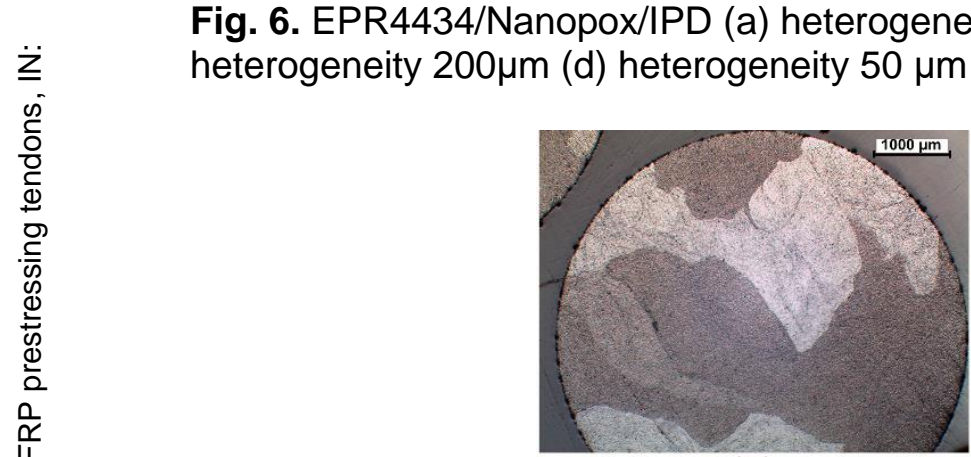

(a)

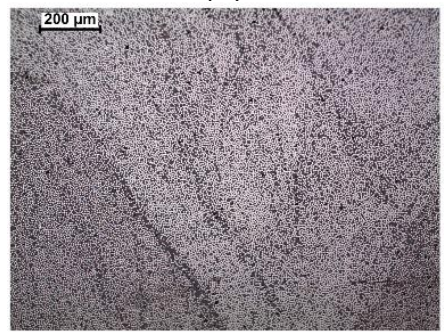

(c)

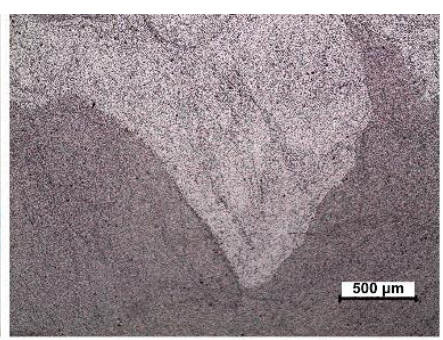

(b)

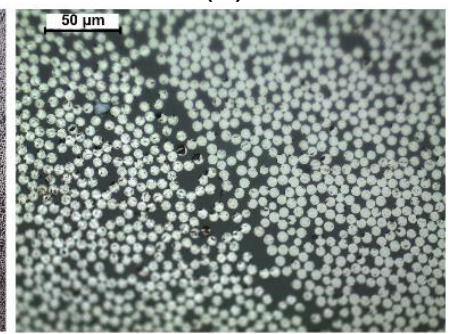

(d)

Fig. 7. Uptake in $5.4 \mathrm{~mm}$ desiccated tendons after exposure to water at either $23^{\circ} \mathrm{C}$ or $60^{\circ} \mathrm{C}$

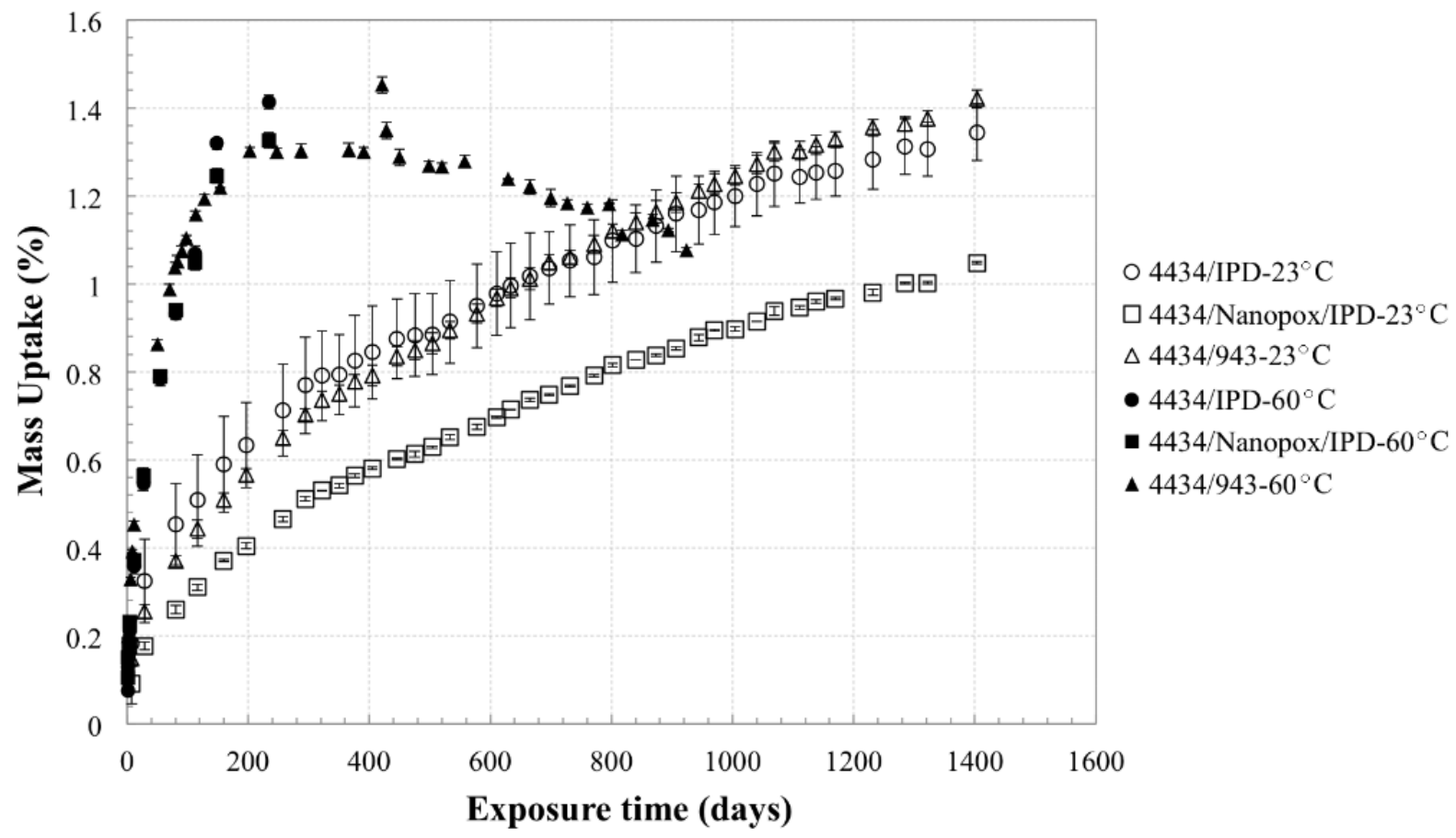


Fig. 8. (a) Schematic progression of moisture uptake, and predicted moisture profiles for (b) 4434/943 (c) 4434/IPD and (d) 4434/Nanopox/IPD tendons after 28, 56 and 127 days of exposure at $23^{\circ} \mathrm{C}$

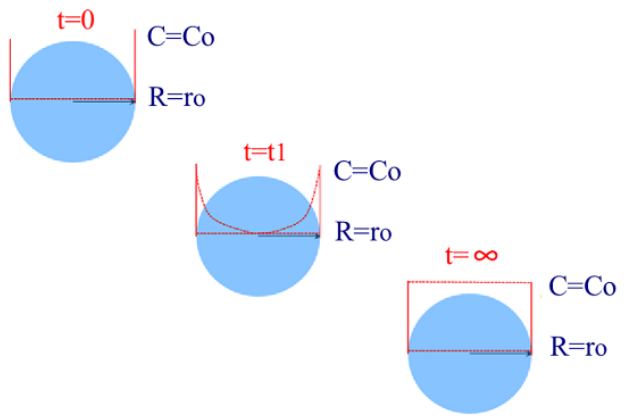

(a)

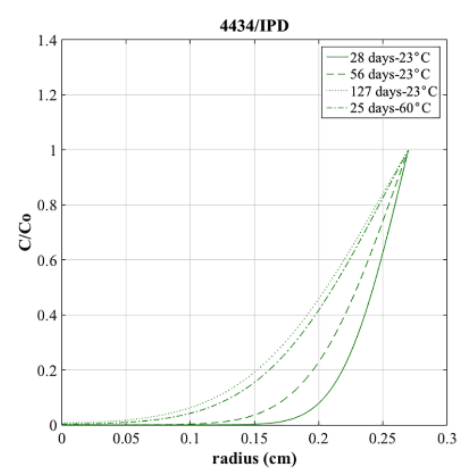

(c)

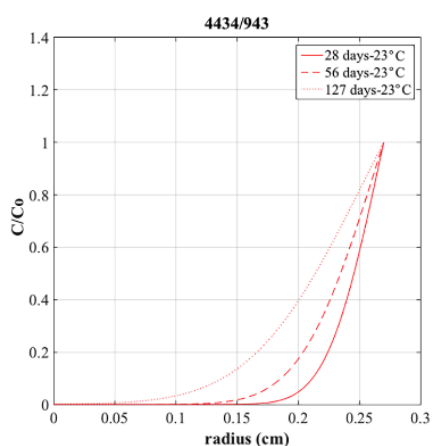

(b)

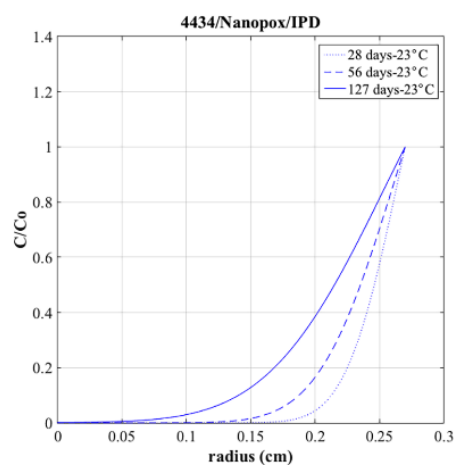

(d)

Fig. 9. Photographs of representative tendon fracture modes after compression test (a) between parallel plates and with-out sand and (b) between saddles and with sand

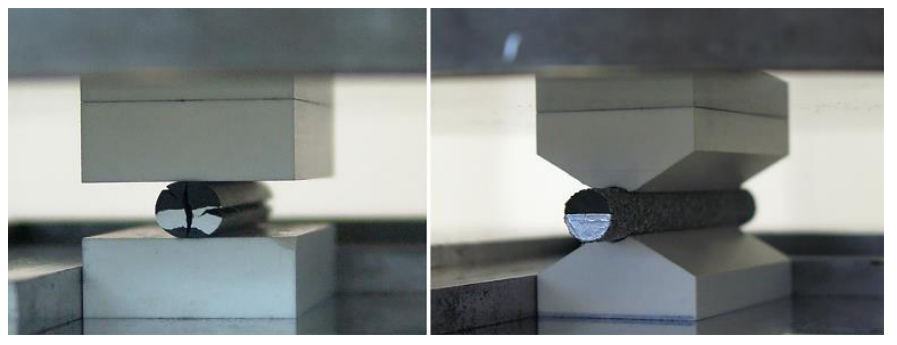

(a)

(b) 
Fig. 10. Sustained loading test - failed CFRP prestressed spun concrete pole after 16.5 years of sustained bending

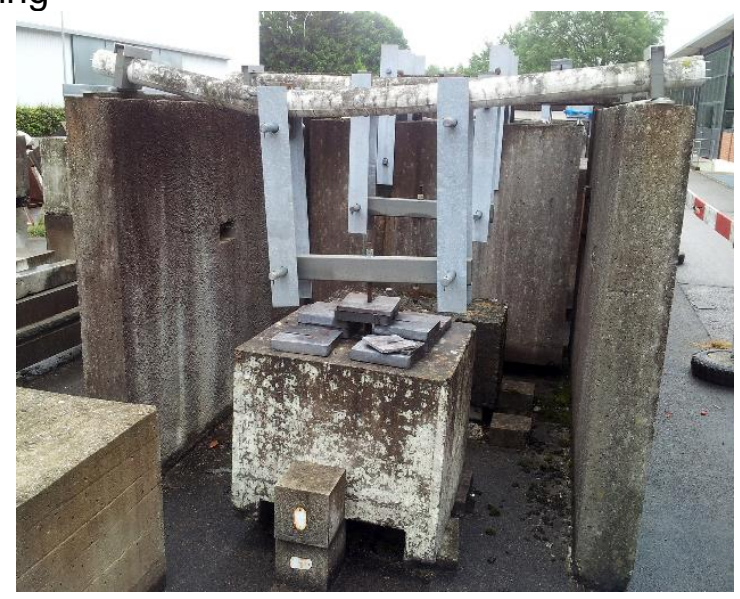

Fig. 11. Tensile test results for aged tendon samples (a) tensile strength and (b) elastic modulus *denotes anchorage failure

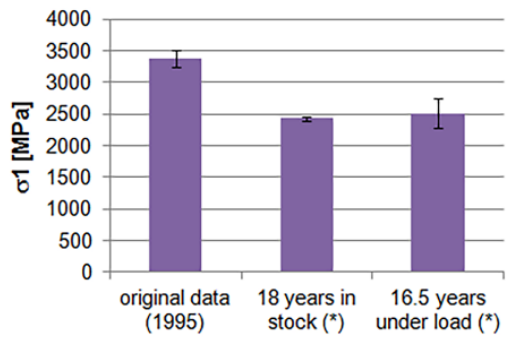

(a)

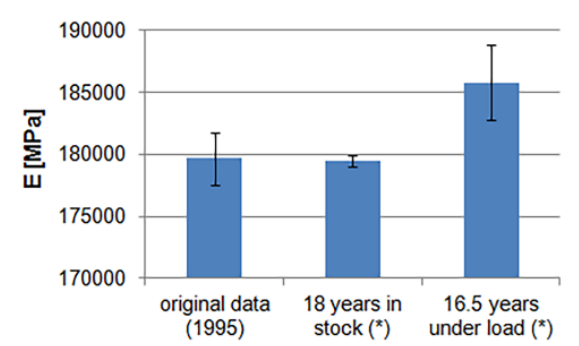

(b) 